\title{
INFLUENCIA DE DOS TIPOS DE CALENTAMIENTO SOBRE LA FUERZA EXPLOSIVA DEL TREN INFERIOR EN ESTUDIANTES DE EDUCACIÓN FÍSICA
}

\section{INFLUENCE OF TWO TYPES OF WARM UP ON THE EXPLOSIVE FORCE OF THE LOWER BODY IN PHYSICAL EDUCATION STUDENTS}

\author{
Alfonso Ruiz Garrigós ${ }^{1}$, Álvaro de Vicente Durán ${ }^{1}$ y Alejandro Sánchez-Pay ${ }^{1}$ \\ ${ }^{1}$ Universidad de Murcia, España \\ alfonso.ruizg@um.es ; alvarode.vicented@um.es ; aspay@um.es \\ Envío Original: 2018-09-29 Reenviado: 2019-12-20 Aceptado: 2020-04-28 \\ Publicado: 2020-05-21
}

Doi: https://doi.org/10.15517/pensarmov.v18i1.34705

\begin{abstract}
Resumen
El objetivo del presente estudio fue determinar las posibles influencias de dos tipos de calentamiento (tradicional y específico) sobre la fuerza explosiva del tren inferior. Se realizaron dos pruebas (salto con contramovimiento -CMJ- y sprint 5 metros) sobre tres grupos: grupo sin calentamiento (GSC), grupo de calentamiento tradicional (GCT) que incluía movilidad articular, carrera continua y estiramientos estáticos; y grupo de calentamiento específico (GCE) que incluía movilidad articular, carrera continua, estiramientos dinámicos y pliometría. Se realizó un pre y post test y se utilizó la prueba de ANOVA de medidas repetidas para comparar la influencia del tipo de calentamiento sobre las pruebas entre los diferentes grupos. Posteriormente se calculó el tamaño del efecto a través de $g$ de Hedges. Los resultados mostraron diferencias estadísticamente significativas entre el pre y post test en el GCT y en el GCE;. además, se encontraron diferencias estadísticamente significativas $(p<.05)$ entre GSC y el GCE en el post test sólo en la prueba de salto. Realizar estiramientos dinámicos y pliometría como parte del calentamiento parece influir positivamente en la capacidad de salto vertical. Los datos sugieren que los profesores de educación física deberían utilizar calentamientos con estiramientos dinámicos y pliometría para mejorar el rendimiento en actividades que incluyan el salto.
\end{abstract}


Palabras clave: estiramiento, calentamiento, CJM, test velocidad.

\begin{abstract}
The aim of the present study was to determine the possible influences of two types of warm-up (traditional and specific) on the explosive strength of the lower train. Two tests were carried out (Counter Movement Jump -CMJ- and 5 meters sprint) in three groups: group not warm-up (GSC), traditional warm-up (GCT) which included articulated mobility, continuous running and static stretching; and specific warm-ups group (GCE) performed a articulated mobility, continuous running, dynamic stretching and plyometric exercises. A pre and post test was performed and ANOVA was used to compare the influence of the type of warm-up on tests and between groups. Subsequently the effect size was calculated through Hedges' g. The results showed statistically significant differences between the pre and post test in the GCT and in the GCE; furthermore, statistically significant differences $(p<.05)$ were found in CMJ test between GSC and GCE, in the posttest. Performing dynamic stretching and plyometric exercises as part of the warm-ups seems to positively influence the ability to jump vertically. The data suggest that teachers of physical education should use warm-ups with dynamic stretching and plyometric exercises to enhance performance in activities that include jump.
\end{abstract}

Key words: stretching, warm-up, CJM, sprint test.

\title{
INTRODUCCIÓN
}

Previo a la actividad deportiva, los deportistas suelen realizar un calentamiento. Por regla general, el calentamiento suele incluir una fase de movilidad articular, carrera continua (activación cardiovascular) y estiramientos; si bien, estas fases dependen de cada autor (Costa, 2009).

En los últimos años, son numerosos los estudios científicos que han estudiado cómo el efecto agudo de la práctica de estiramientos repercute en el rendimiento deportivo, observándose, en varias ocasiones, controversias en los resultados (Rubini, Costa y Gomes, 2007). Por un lado, existen autores que afirman que los estiramientos estáticos no afectan de ninguna manera al rendimiento; es decir, no aportan beneficios ni inconvenientes que influyan en el tiempo de reacción o en la fuerza explosiva del movimiento (Alpkaya y Koceja, 2007). Por el contrario, existe otra corriente de autores que 
sugieren que el estiramiento muscular influye de forma negativa al rendimiento si se usa previo a la máxima contracción voluntaria (Behm, Bradbury, Haynes, Hodder, Leonard y Paddock, 2006; Cramer, et al., 2007; Fowles Sale y Macdougall, 2000; Marek, et al., 2005; Ogura, Miyahara, Naito, Katamoto y Auki, 2007; Power, Behm, Cahill, Carroll y Young, 2004; Yamaguchi, Ishii, Yamanaka y Yasuda, 2006). De igual forma, el estiramiento muscular podría influir negativamente en la altura alcanzada en un salto vertical (Carvalho, et al., 2012; Church, Wiggins, Moode y Crist, 2001; Cornwell, Nelson y Sidaway 2002; Young y Behm, 2002) o la velocidad de carrera en un sprint (Fletcher y Anness, 2007; Fletcher y Jones, 2004; Little y Williams, 2006; Nelson, Driscoll, Landin, Young y Schexnayder, 2005).

Los resultados que sugiere la evidencia anteriormente comentada podrían tener relación con determinadas tendencias que determinan que los estiramientos estáticos incluidos en el calentamiento previo a una actividad explosiva (con implicación notoria de la potencia muscular, y con ello el Ciclo Estiramiento Acortamiento (CEA) pueden repercutir de forma negativa al rendimiento deportivo (Woods, Bishop y Jones, 2007). Así, algunos estudios han demostrado que el estiramiento estático tiene una influencia negativa sobre la fuerza explosiva en un salto vertical, mientras que el estiramiento dinámico posee un impacto positivo. Esto podría ser atribuido un posible deterioro neurológico y una posible alteración en las propiedades visco elásticas de las unidades del tendón muscular (Hough, Ross y Howatson, 2009).

En un calentamiento donde estén presentes los estiramientos dinámicos frente a los estiramientos estáticos, puede que mejore el rendimiento deportivo, debido al efecto postactivación potenciación (PAP), generado en el músculo (Freitas, Andrade, Larcoupaille, Mil-Homens y Nordez, 2015). Así, estos autores encontraron que los efectos de estiramiento en la articulación y en el músculo dependen de su intensidad, ya que el estiramiento al 40 o $60 \%$ del rango de movimiento de la dorsiflexión máxima no afecta al módulo elástico, pero sí parece afectar a intensidades del $80 \%$ del rango de movimiento de la dorsiflexión máxima.

El calentamiento puede incluir ejercicios/tareas donde el CEA en la musculatura esté presente. En el estudio desarrollado por Palao, Saenz y Ureña (2001) encontraron que ejercicios de técnica de carrera de la musculatura flexo-extensora con participación del CEA, conlleva a la mejora de acciones técnicas específicas de salto en voleibol (Palao, et al., 2001). Este hallazgo nos demuestra que, mediante la inclusión en el calentamiento de ejercicios que focalicen el CEA, se mejora la potencia en el salto. 
La utilización de calentamientos donde estén presentes estiramientos dinámicos así como ejercicios de pliometría, podría influir en las acciones explosivas (saltar y esprintar) que se dan en una clase de Educación Física dentro de los contenidos de específicos de multitud de deportes. Por lo tanto, el objetivo del presente estudio fue analizar la posible influencia de dos tipos de calentamiento sobre los niveles de fuerza explosiva en las pruebas salto con contramovimiento (CMJ) y sprint de 5 metros en estudiantes de Educación Física.

\section{MÉTODO}

\section{Participantes}

La muestra objeto de estudio estuvo compuesta por 27 adolescentes (10 mujeres y 17 hombres) con una edad media de $16.48 \pm 0.68$ años, $64.04 \pm 8.77 \mathrm{~kg}$ de peso, y $1.71 \pm$ $0.09 \mathrm{~cm}$ de talla. Todos los sujetos eran estudiantes pre-universitarios que cursaban la asignatura de Educación Física. Todos los sujetos fueron informados de las características del estudio y se contó con el consentimiento informado de los tutores de los mismos así como del centro educativo.

\section{Procedimiento}

La muestra fue dividida en tres grupos de forma aleatoria. Un grupo no realizó calentamiento entre el pre-test y el post-test. Los otros dos grupos realizaron un calentamiento guiado con una duración total aproximada de 6-7 minutos (ver tabla 1). 
Tabla 1.

Descripción de los ejercicios realizados en cada calentamiento

\begin{tabular}{|c|c|}
\hline $\begin{array}{l}\text { Grupo de calentamiento tradicional } \\
\text { GCT }\end{array}$ & $\begin{array}{l}\text { Grupo de calentamiento específico } \\
\text { GCE }\end{array}$ \\
\hline $\begin{array}{l}\text { Movilidad articular } \text { general: } \\
\text { circunducción de tobillo (10" cada uno), } \\
\text { flexo-extensión de rodilla en estático } \\
(15 ") \text {, rotación de cadera (15"), rotación } \\
\text { de hombro hacia adelante y hacia atrás } \\
\text { (10" cada uno), movimientos laterales } \\
\text { alternativos de cuello (10"). }\end{array}$ & $\begin{array}{l}\text { Carrera continua con movilidad articular } \\
\text { dinámica ( } 4 \text { minutos): ( } 30 ") \text { de carrera } \\
\text { normal, (15") de elevación de rodillas, } \\
(15 ") \text { de talones atrás, (15") de flexión de } \\
\text { cadera, (15") de hiperextensión de } \\
\text { cadera con inclinación de tronco hacia } \\
\text { adelante (15"), rotación externa de } \\
\text { cadera (15"), rotación interna de cadera }\end{array}$ \\
\hline $\begin{array}{l}\text { Carrera continua a } \\
\text { minutos). }\end{array}$ & $\begin{array}{l}\text { ), flexo- } \\
\text { ra hasta }\end{array}$ \\
\hline
\end{tabular}

Fase de flexibilidad general: estiramiento de isquiosurales sentados (15" cada pierna), estiramiento de cuádriceps apoyados en pared o compañero (15" cada pierna), estiramiento de psoas ilíaco en posición de zancada (15" cada pierna), estiramiento de pectoral en pared o con compañero (10" cada brazo), estiramiento del dorsal ancho en pared o en hombros de un compañero (15").

Fase de flexibilidad dinámica: movimiento de flexión y extensión de rodilla aumentando la amplitud de movimiento progresivamente (15" cada pierna), movimiento de flexión e hiperextensión de cadera aumentando la amplitud de forma progresiva (15" cada pierna), abducción y aducción de cadera aumentando la amplitud de forma progresiva (15" cada pierna), zancadas dinámicas enfatizando el estiramiento en el psoas ilíaco (30").

Pliometría: desde una posición elevada (banco sueco) los sujetos se dejan caer con un ligero ángulo hacia adelante y recepcionarán con la punta de los pies, realizando un salto con el mínimo tiempo de contacto de los pies con el suelo. Se realizarán 3-5 saltos.

\section{Fuente: elaboración propia}

Cada grupo realizó dos pruebas (test de salto y test de sprint de 5 metros) antes y después de cada calentamiento. Primeramente, todos los estudiantes realizaron el test de salto y de sprint de forma conjunta (distribuido en dos estaciones de medición). Posteriormente, el GCT realizó de forma conjunta los ejercicios expuestos en la tabla 1 como calentamiento. Tras ello, volvieron a realizar los test de salto y sprint de 5 metros. Tras finalizar los test del GCT, el GCE realizó los ejercicios descritos en la tabla 1 para 
seguidamente realizar nuevamente los test de salto y sprint de 5 metros. Por último, el GSC realizó el test nuevamente.

Test de salto CMJ: se realizó un salto con contramovimiento por cada participante. El sujeto partió desde una posición erguida y con las manos en las caderas. Se le pidió que realizase un salto hacia arriba por medio de una flexión seguida lo más rápidamente de una extensión de piernas. La flexión debía llegar hasta un ángulo de 90 grados. Las piernas durante la fase de vuelo tenían que estar extendidas y mantener esa extensión hasta el momento de contacto con el suelo (Bosco, 1994).

Sprint de 5 metros: Los sujetos, en posición de salida alta y sin apoyo de manos, se posicionaron detrás de la línea de salida con un pie adelantado al otro. A la señal visual del controlador (pulgar hacia arriba) el ejecutante tenía que recorrer la distancia de 5 metros en el menor tiempo posible.

Ambas pruebas fueron filmadas con un dispositivo móvil (Samsung Galaxy S8, Seoul, South Korea). Las pruebas fueron grabadas a cámara lenta (240 fotogramas . segundo $^{-1}$ ) tal y como se ha realizado en otros estudios (Bond, Willaert y Noonan, 2017; García-López, Peleteiro, Rodríguez-Marroyo, Morante, Herrero y Villa, 2005). Para la prueba de sprint de 5 metros, el dispositivo se situó perpendicular a la línea de carrera y a 5 metros de ésta. Para la prueba de salto, el dispositivo se situó en la línea del suelo para ver con mayor precisión el contacto de los pies. Los videos fueron analizados en el Software gratuito Kinovea 0.8.25 (Francia) para Windows. Un observador experimentado registró el tiempo en milésimas de segundo en cada prueba: Test de salto, desde el primer fotograma en el cual ninguno de los pies está en contacto con el suelo, hasta el fotograma en el cual alguno de los pies toma contacto con el suelo. Sprint de 5 metros, desde el primer fotograma en el cual se veía la señal visual del controlador, hasta el fotograma en el que el pecho cruza la línea de la llegada.

\section{Análisis estadístico}

Tras la toma de datos se realizó un análisis estadístico a través del paquete estadístico SPSS 21.0 para Mac. Se llevó a cabo un análisis descriptivo presentando los valores medios y desviación típica de cada variable. El tamaño de la muestra recomendó el uso de Shapiro Wilk para contrastar la normalidad de las variables. Adicionalmente, se realizó ANOVA de 1 vía de grupos independientes (3 grupos) para comparar la homogeneidad entre grupos en el pre-test. Además, de ser necesario, se realizaron pruebas post hoc con el ajuste de Bonferroni para identificar las diferencias 
estadísticamente significativas. Tal y como se ha realizado en otros estudios (Hernández, et al., 2018), para analizar la diferencia entre variables (salto y sprint) en función del tipo de calentamiento empleado se utilizó la prueba ANOVA de 3 grupos x 2 mediciones (prepost). Además, se calculó el tamaño del efecto en las variables usando el método de la desviación típica ponderada de los dos momentos (pre y post). Se calculó el tamaño del efecto a través de la g de Hedges debido al tamaño muestral (Hedges y Olkin, 1985). Para determinar la magnitud del efecto se siguieron las recomendaciones de Rhea (2004) para deportistas no entrenados, siendo <0.50 considerado como trivial; 0.50-0.1.25 considerado como bajo; 1.25-1.9 moderado; y > 2.0 considerado como grande. El tamaño del efecto y el análisis de varianza se realizó a través de la hoja de cálculo jamovi 1.2.2 basada en el gráfico interfaz de usuario R. La significación se estableció en p <.05.

\section{RESULTADOS}

En la tabla 2 se observan los valores medios y desviación típica de las pruebas de salto y sprint en los diferentes tipos de calentamiento. La prueba de Shapiro-Wilk mostró normalidad de los datos tanto en el salto ( $p r e: p=0.312$, y post: $p=0.175$ ) como en el sprint de 5 metros (pre: $p=0.275$, y post: $p=0.084$ ). El ANOVA de una vía de grupos independientes (3 grupos) mostró la homogeneidad de los grupos en las pruebas de salto y sprint, no encontrándose diferencias estadísticamente significativas entre los tres grupos en las pruebas pre test (salto, $p=0.454$; sprint, $p=0.292$ ).

Tabla 2.

Análisis descriptivo y prueba de homogeneidad en las variables del test CMJ y Sprint 5 metros en función de los diferentes tipos de calentamiento.

\begin{tabular}{llccccc}
\hline & & GSC & GCT & GCE & $p$ & F \\
\hline \multirow{3}{*}{ Test de salto } & Pre & $0.412 \pm .08$ & $0.411 \pm .07$ & $0.450 \pm .05$ & 0.454 & 1.010 \\
& Post & $0.424 \pm .07$ & $0.467 \pm .07$ & $0.511 \pm .05$ & & \\
\cline { 2 - 7 } Test de sprint & Pre & $1.811 \pm .22$ & $1.848 \pm .17$ & $1.750 \pm .13$ & 0.292 & 0.822 \\
& Post & $1.792 \pm .24$ & $1806 \pm .20$ & $1.672 \pm .15$ & & \\
\hline
\end{tabular}

Nota: GSC: Grupo sin calentamiento. GCT: Grupo con calentamiento tradicional. GCE: Grupo con calentamiento específico. p: Significación. Fuente: elaboración propia.

El ANOVA de dos vías mixto de 3 grupos $x 2$ mediciones (pre-post) mostró diferencias estadísticamente significativas ( $p=0.032, F=3.98$ ), en el salto (figura 1 ). El 
análisis post hoc reveló diferencias estadísticamente significativas entre el pre-post del GCE ( $p=0.002 ; g=1.22)$ así como en el pre-post del GCT ( $p=0.004 ; g=0.76)$. Además, se encontraron diferencias estadísticamente significativas entre el GCE y el GSC en el post test $(p=0.045 ; g=1.40)$.

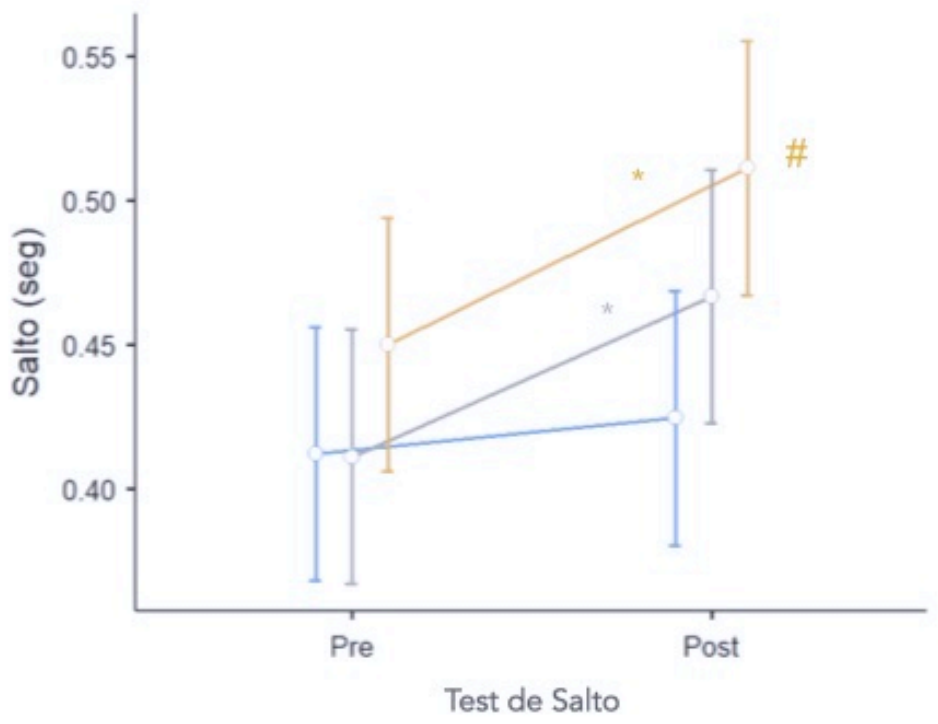

\section{Calentamiento}

Sin calentamiento

Calentamiento tradicional

Calentamiento especifico

Figura 1. Variación de los valores medios en la prueba de salto en los grupos sin calentamiento (GSC), grupo con calentamiento tradicional (GCT) y grupo con calentamiento específico (GCE). Nota: ${ }^{*}=p<0.05$ Pre-Post. \# $=p<0.05$ GSC-GCE. Fuente: elaboración propia

EI ANOVA de dos vías mixto, 3 grupos $\times 2$ mediciones (pre-post) no mostró diferencias estadísticamente significativas en el sprint ( $p=0.499, F=0.717$, ver figura 2). Por tanto, en congruencia con lo anterior, el análisis post hoc tampoco reveló diferencias estadísticamente significativas entre el pre-post en ninguno de los grupos (GSC $p=0.994$, $g=0.08 ;$ GCT $p=0.669, g=0.27 ;$ GCE $p=0.261, g=0.52$ ). 


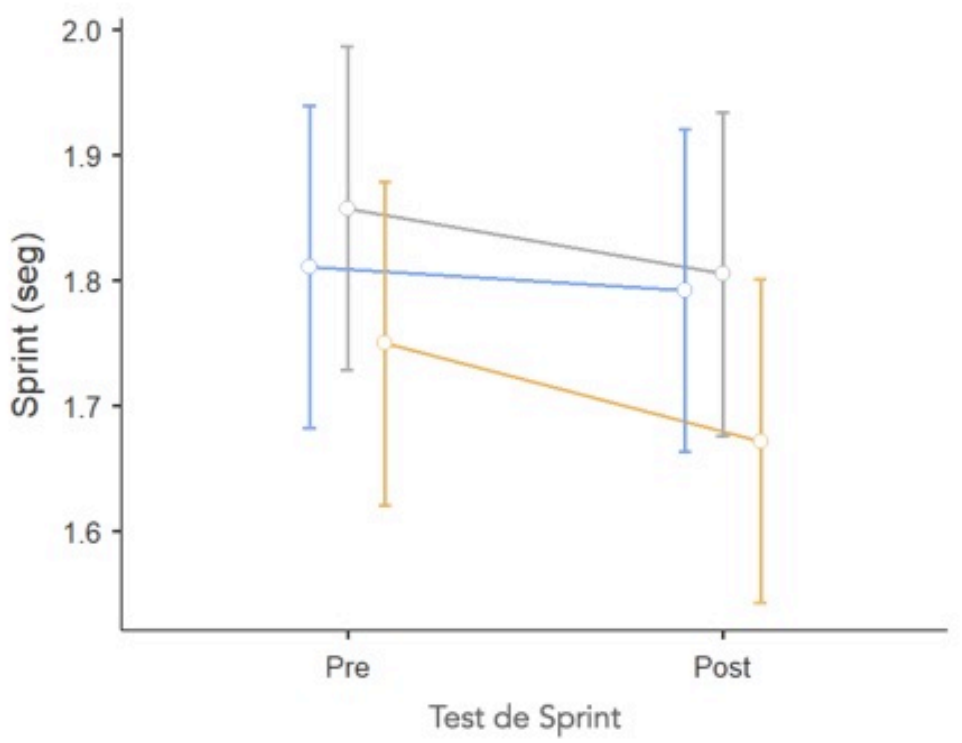

Calentamiento

Sin calentamiento

Calentamiento tradicional

Calentamiento especifico

Figura 2. Variación de los valores medios en la prueba de sprint en los grupos sin calentamiento (GSC), grupo con calentamiento tradicional (GCT) y grupo con calentamiento específico (GCE). Fuente: elaboración propia.

\section{DISCUSIÓN}

Analizar la influencia de las características del calentamiento sobre una actividad explosiva es de vital importancia para desarrollar tareas acordes a los objetivos planteados. Los resultados de este estudio muestran cómo un calentamiento consistente en estiramientos dinámicos y ejercicios de pliometría, influye positivamente sobre la acción explosiva de salto si se compara con la ausencia de un calentamiento previo o un calentamiento tradicional.

El análisis pre-post mostró diferencias estadísticamente significativas tanto en el GCT y en el GCE sólo en la prueba de salto (figura 1). Los estudiantes, tanto con un calentamiento tradicional como con uno específico, mejoraron los valores de salto tras el mismo. El estiramiento estático parece disminuir la capacidad de salto, no así con el estiramiento dinámico (Carvalho et al., 2012; Hough et al., 2009). En este estudio, las mejoras en el GCT podrían deberse, por un lado, a que no sólo se incluye el estiramiento como parte del calentamiento; por otro, la corta duración del estiramiento comparado con otros estudios.

Los datos de este estudio muestran diferencias estadísticamente significativas en el salto post entre los grupos $(p<.05)$. Aunque parezca existir una tendencia en la disminución (en el sprint) y un aumento (en el salto) de los valores en el GSC (tabla 1), 
esto puede ser debido a que la práctica de la propia prueba (pre-test) influya en la mejora de la misma a causa de su repetición (como parte de un calentamiento, o aprendizaje técnico de la misma). La comparación entre grupos en el post test muestra que existen diferencias estadísticamente significativas con la comparación entre el GSC y el GCE en la prueba de salto (figura 1). Las mejoras en los valores tras realizar un calentamiento específico siguen la línea de otros estudios en jugadores de fútbol (Ayala y Sainz de Baranda, 2010) futbolistas universitarios (Jerez-Mayorga, Contreras-Díaz y Campos-Jara, $\underline{2013}$ ) o tenistas junior de élite (Ayala, Moreno-Pérez, Vera-García, Moya, Sanz-Rivas y Fernández Fernández, 2016); si bien, todos ellos utilizaban el sprint de 20 metros, otros utilizaban tiempos de calentamiento cercanos a 30 minutos (Ayala et al., 2016), aspecto que no puede ser considerado en el contexto escolar debido a la limitación de tiempo. Las diferencias entre el GSC y el GCE en la prueba de salto mostraron diferencias estadísticamente significativas $(p<0.05)$ y un tamaño del efecto considerado moderado para personas no entrenadas $(g=1.40)$. Las diferencias representan una mejora superior al $10 \%$ en la prueba de salto si comparamos entre grupos. Esta diferencia es muy superior a la encontrada por Thompsen, Kackley, Palumbo, y Faigenbaum (2007) donde los sujetos mostraron una mejora cercana al $5 \%$ tanto en la altura de salto vertical como en la distancia de salto horizontal tras aplicar una rutina de estiramientos dinámicos del tren inferior. Estas diferencias podrían explicarse debido a los niveles iniciales de los deportistas (estudiantes escolares y deportistas de alto nivel), así como la diferencia en los protocolos de medición usados en ambos estudios.

El calentamiento es una parte fundamental dentro de cualquier actividad deportiva realizándose igualmente dentro de las sesiones de EF (Devís y Velert, 1992). Este calentamiento suele incluir estiramientos, cuyos diferentes tipos, han suscitado controversia en lo que a mejoras en el rendimiento se refiere (Alpkaya y Koceja, 2007; Ogura, et al., 2007). Los datos encontrados en este estudio indican que tanto un calentamiento tradicional (con estiramientos estáticos) como un calentamiento específico (con estiramientos dinámicos) mejoran el rendimiento de fuerza explosiva de salto en estudiantes de EF. Si bien, la mejora parece ser mayor con estiramientos dinámicos si comparamos los tamaños del efecto (0.76 vs 1.22). En este sentido, el uso de los estiramientos dinámicos puede ser recomendable en las sesiones de EF que incluyan actividades explosivas específicas. De esta forma, los profesores de EF podrían incluir estiramientos dinámicos dentro del calentamiento antes de la práctica de deportes como el voleibol, baloncesto o balonmano, donde la acción de salto es muy común. 
Este estudio posee una serie de limitaciones que deben ser tenidas en cuenta. Por un lado, los deportistas no estaban familiarizados con las pruebas, lo que puede dar lugar a errores en la ejecución de los saltos. Además, la grabación a 240 fps muestra un error de la medida de $\pm 0,004$ segundos entre cada fotograma. En la actualidad, los instrumentos de laboratorio que se encargan de medir los tiempos de vuelo (plataformas de contacto) o los tiempos de carrera (fotocélulas) suelen tener un margen de error de \pm 0,001 segundos, aspecto que debe ser tenido en consideración.

Tal y como apunta Ayala et al. (2011), se hace necesario realizar un análisis de los diferentes componentes de la rutina de estiramientos en términos de duración, técnica o intensidad de los mismos, pues podría permitir una mejor comprensión del efecto agudo real del estiramiento sobre el rendimiento deportivo. 


\section{REFERENCIAS}

Alpkaya, U. y Koceja, D. (2007). The effects of acute static stretching on reaction time and force. Journal of Sports Medicine and Physical Fitness, 47(2), 147-150. Recuperado de: https://www.ncbi.nlm.nih.gov/pubmed/17557051

Ayala, F., Moreno-Pérez, V., Vera-García, F. J., Moya,. M., Sanz-Rivas, D. y FernandezFernandez, J. (2016). Acute and time-course effects of traditional and dynamic warm-up routines in young elite junior tennis players. Plos One, 11(4), e0152790. doi: https://doi.org/10.1371/journal.pone.0152790

Ayala, F. y Sainz de Baranda, P. (2010). Efecto agudo del estiramiento sobre el sprint en jugadores de fútbol de división de honor juvenil. RICYDE. Revista Internacional de Ciencias del Deporte, 6(18), 1-12. Recuperado de: http://www.redalyc.org/articulo.oa?id=71012449002

Ayala, F., Sainz, P., Cejudo, A. y de Ste Croix, M. (2011). Efecto agudo del estiramiento sobre el rendimiento físico: el uso de los estiramientos en el calentamiento. Cultura Ciencia Deporte, 6(16), 27-36. doi: http://dx.doi.org/10.12800/ccd.v6i16.31

Behm, D. G., Bradbury, E. E., Haynes, A. T., Hodder, J. N., Leonard, A. M. y Paddock, N. R. (2006). Flexibility is not related to stretch-induced deficits in force or power. Journal of Sports Science \& Medicine, 5(1), 33-42. Recuperado de: https://www.ncbi.nlm.nih.gov/pubmed/24198679

Bond, C. W., Willaert, E. M., y Noonan, B. C. (2017). Comparison of three timing systems: reliability and best practice recommendations in timing short-duration sprints. Journal of Strength and Conditioning Research, 31(4), 1062-1071. doi: https://doi.org/10.1519/JSC.0000000000001566

Bosco, C. (1994). La valoración de la fuerza con el test de Bosco. Barcelona: Paidotribo.

Carvalho, F. L., Carvalho, M. C., Simão, R., Gomes, T. M., Costa, P. B., Neto, L. B., ... y Dantas, E. H. (2012). Acute effects of a warm-up including active, passive, and dynamic stretching on vertical jump performance. The Journal of Strength \& Conditioning Research, 26(9), 2447-2452. doi: https://doi.org/10.1519/JSC.0b013e31823f2b36

Church, B.J., Wiggins, M.S., Moode, M.F. y Crist, R. (2001). Effect of warm-up and flexibility treatments on vertical jump performance. Journal of Strength and Conditioning Research, 15(3), 332-336. Recuperado de: https://www.ncbi.nlm.nih.gov/pubmed/11710660

Costa, J. (2009). El calentamiento general y específico en la educación Física. Ejercicios prácticos. Cultivalibros. Recuperado de https://www.libreriadeportiva.com/libro/elcalentamiento-general-y-especifico-en-la-educacion-fisica-ejerciciospracticos 30952

Cornwell, A., Nelson, A.G. y Sidaway, B. (2002). Acute effects of stretching on the neuromechanical properties of the triceps surae muscle complex. European Journal of Applied Physiologic, 86(5), 428-434. doi: https://doi.org/10.1007/s00421$\underline{001-0565-1}$ 
Cramer, J.T., Beck, T.W., Housh, T.J., Massey, L.L., Marek, S.M., Danglemeier, S., Purkayastha, S., .... y Egan, A. (2007). Acute effects of static stretching on characteristics of the isokinetic angle-torque relationship, surface electromyography, and mechanomyography. Journal of Sports Sciences, 25(6), 687-698. doi: https://doi.org/10.1080/02640410600818416

Devís, J. D. y Velert, C. P. (1992). Nuevas perspectivas curriculares en educación física: la salud y los juegos modificados (Vol. 103). Barcelona: Inde. Recuperado de https://www.inde.com/es/productos/detail/pro id/45

Fletcher, I. M. y Jones, B. (2004). The effect of different warm-up stretch protocols on 20 meter sprint performance in trained rugby union players. The Journal of Strength \& Conditioning Research, 18(4), 885-888. doi: https://doi.org/10.1519/14493.1

Fletcher, I. y Anness, R. (2007). The acute effects of combined static and dynamic stretch protocols on fifty-meter sprint performance in track-and-field athletes. Journal of Strength and Conditioning Research, 21(3), 784-787. doi: https://doi.org/10.1519/R-19475.1

Fowles, J.R., Sale, D.G. y MacDougall, J.D. (2000). Reduced strength after passive stretch of the human plantarflexors. Journal of Applied Physiologic, 89(3), 1179-1188. doi: https://doi.org/10.1152/jappl.2000.89.3.1179

Freitas, S. R., Andrade, R. J., Larcoupaille, L., Mil-homens, P. y Nordez, A. (2015). Muscle and joint responses during and after static stretching performed at different intensities. European Journal of Applied Physiology, 115(6), 1263-1272. doi: https://doi.org/10.1007/s00421-015-3104-1

García-López, J., Peleteiro, J., Rodríguez-Marroyo, J. A., Morante, J. C., Herrero, J. A. y Villa, J. G. (2005). The validation of a new method that measures contact and flight times during vertical jump. International Journal of Sports Medicine, 26(4), 294-302. doi: https://doi.org/10.1055/s-2004-820962

Hedges L. V., y Olkin I. (1985). Statistical methods for meta-analysis. San Diego CA: Academic Press. Recuperado de https://www.elsevier.com/books/statisticalmethods-for-meta-analysis/hedges/978-0-08-057065-5

Hernández, S., Ramirez-Campillo, R., Álvarez, C., Sanchez-Sanchez, J., Moran, J., ... y Loturco, I. (2018). Effects of plyometric training on neuromuscular performance in youth basketball players: a pilot study on the influence of drill randomization. Journal of Sports Science and Medicine, 17(3), 372-378. Recuperado de https://www.ncbi.nlm.nih.gov/pubmed/30116110

Hough, P. A., Ross, E. Z., y Howatson, G. (2009). Effects of dynamic and static stretching on vertical jump performance and electromyographic activity. The Journal of Strength \& Conditioning Research, 23(2), 507-512. Recuperado de https://www.ncbi.nlm.nih.gov/pubmed/19204571

Jerez-Mayorga, D., Contreras-Díaz, G. y Campos-Jara, C. (2013). Efectos agudos de diferentes protocolos de estiramiento en la capacidad de salto vertical y sprint en futbolistas universitarios. Revista Horizonte Ciencias de la Actividad Física, 4(2), 105-117.

Recuperado

de: http://revistahorizonte.ulagos.cl/index.php/horizonte/article/view/52 
Little, T. y Williams, A.G. (2006). Effects of differential stretching protocols during warmups on high-speed motor capacities in professional soccer players. Journal of Strength and Conditioning Research, 20(1), 203-207. doi: https://doi.org/10.1519/R-16944.1

Marek, S.M., Cramer, J.T., Fincher, A.L., Massey, L.L., Dangelmater, S.M., Purkayastha, S., ... y Culbertson, J.Y. (2005). Acute effects of static and propioceptive neuromuscular facilitation stretching on muscle strength and power output. Journal of Athletic Training, 40(2), 94-103. Recuperado de: https://www.ncbi.nlm.nih.gov/pmc/articles/PMC1150232/

Nelson, A. G., Driscoll, N. M., Landin, D. K., Young, M. A. y Schexnayder, I. C. (2005). Acute effects of passive muscle stretching on sprint performance. Journal of Sports Science, 23(5), 449-454. doi: https://doi.org/10.1080/02640410410001730205

Ogura, Y., Miyahara, Y., Naito, H., Katamoto, S. y Aoki, J. (2007). Duration of static stretching influences muscle force production in hamstring muscles. Journal of Strength and Conditioning Research, 21(3), 788-792. Recuperado de https://europepmc.org/article/med/17685679

Palao, J.M., Saenz, B. y Ureña, A. (2001). Efecto de un trabajo de aprendizaje del ciclo estiramiento-acortamiento sobre la capacidad de salto en voleibol. Revista Internacional de Medicina y Ciencias de la Actividad Física y el Deporte, 1(3), 163176. Recuperado de: http://cdeporte.rediris.es/revista/revista3/artvolei.html

Power, K., Behm, D., Cahill, F., Carroll, M. y Young, W. (2004). An acute bout of static stretching: effects on force and jumping performance. Medicine and Science in Sports and Exercise, 36(8), 1389-1396. Recuperado de: https://www.ncbi.nlm.nih.gov/pubmed/15292748

Rhea, M. R. (2004). Determining the magnitude of treatment effects in strength training research through the use of the effect size. Journal of strength and conditioning research, 18(4), 918-920. doi: https://doi.org/10.1519/14403.1

Rubini, E. C., Costa, A. L. y Gomes, P. S. (2007). The effects of stretching on strength performance. Sports Medicine, 37(3), 213-224. doi: https://doi.org/10.2165/00007256-200737030-00003

Thompsen, A.G., Kackley, T., Palumbo, M. A. y Faigenbaum, A.D. (2007). Acute effects of different warm-up protocols with and without a weighted vest ton dumping performance in athletic women. Journal of Strength and Conditioning Research, 21(1), 52-56. doi: https://doi.org/ https://doi.org/10.1519/00124278-200702000$\underline{00010}$

Woods, K., Bishop, P. y Jones, E. (2007). Warm-up and stretching in the prevention of muscular injury. Sports Medicine, 37(12), 1089-1099. Recuperado de: https://www.ncbi.nlm.nih.gov/pubmed/18027995

Yamaguchi, T., Ishii, K., Yamanaka, M. y Yasuda, K. (2006). Acute effect of static stretching on power output during concentric dynamic constant external resistance leg extension. Journal of Strength and Conditioning Research, 20(4), 804-810. doi: https://doi.org/10.1519/R-18715.1 
Young, W. B. y Behm, D. G. (2002). Should static stretching be used during a warm up for strength and power activities? Journal of Strength and Conditioning, 24(6), 33-37. doi: https://doi.org/10.1519/00126548-200212000-00006 